\title{
On the sighting of unicorns: A variational approach to computing invariant sets in dynamical systems
}

Oliver Junge, and loannis G. Kevrekidis

Citation: Chaos 27, 063102 (2017); doi: 10.1063/1.4983468

View online: $\mathrm{http}: / / \mathrm{dx}$.doi.org/10.1063/1.4983468

View Table of Contents: http://aip.scitation.org/toc/cha/27/6

Published by the American Institute of Physics

\section{Articles you may be interested in}

Complex behavior in chains of nonlinear oscillators

Chaos: An Interdisciplinary Journal of Nonlinear Science 27, 063104 (2017); 10.1063/1.4984800

Revival of oscillations from deaths in diffusively coupled nonlinear systems: Theory and experiment

Chaos: An Interdisciplinary Journal of Nonlinear Science 27, 061101 (2017); 10.1063/1.4984927

Reduced-order description of transient instabilities and computation of finite-time Lyapunov exponents

Chaos: An Interdisciplinary Journal of Nonlinear Science 27, 063103 (2017); 10.1063/1.4984627

Behavioral synchronization induced by epidemic spread in complex networks

Chaos: An Interdisciplinary Journal of Nonlinear Science 27, 063101 (2017); 10.1063/1.4984217

Temporal intermittency and the lifetime of chimera states in ensembles of nonlocally coupled chaotic oscillators Chaos: An Interdisciplinary Journal of Nonlinear Science 27, 061102 (2017); 10.1063/1.4985143

Frequency and phase synchronization in large groups: Low dimensional description of synchronized clapping, firefly flashing, and cricket chirping

Chaos: An Interdisciplinary Journal of Nonlinear Science 27, 051101 (2017); 10.1063/1.4983470

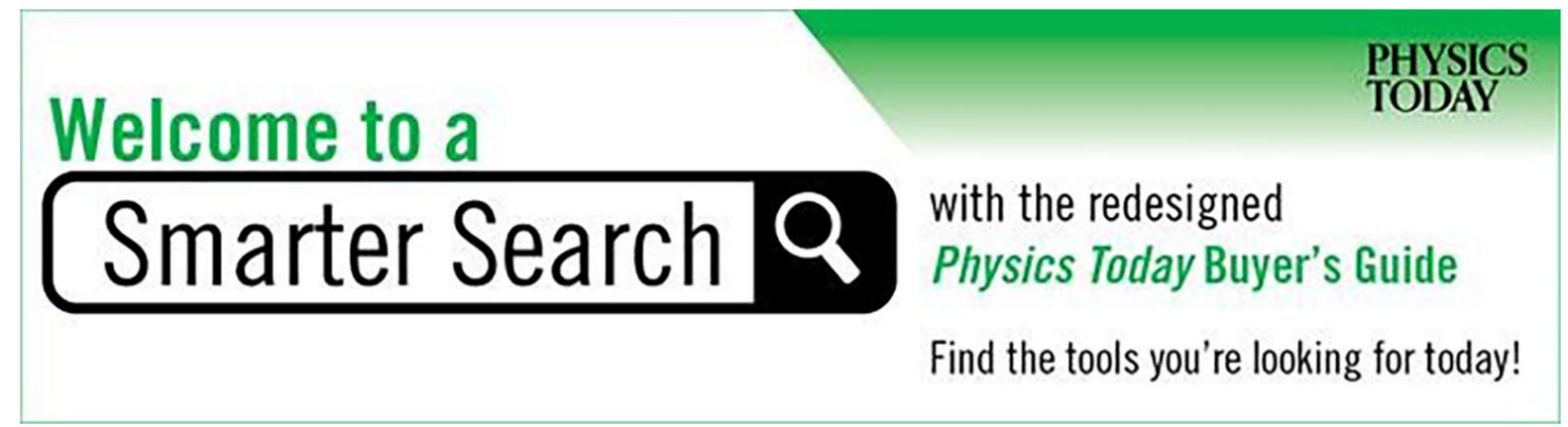




\title{
On the sighting of unicorns: A variational approach to computing invariant sets in dynamical systems
}

\author{
Oliver Junge ${ }^{1, a)}$ and loannis G. Kevrekidis ${ }^{2, b)}$ \\ ${ }^{1}$ Center for Mathematics, Technical University of Munich, 85747 Garching, Germany \\ ${ }^{2}$ Department of Chemical and Biological Engineering and PACM, Princeton University, Princeton, New \\ Jersey 08544, USA and Institute of Advanced Studies, TU Munich, and ZIB, FU Berlin, Berlin, Germany
}

(Received 17 October 2016; accepted 1 May 2017; published online 6 June 2017)

\begin{abstract}
We propose to compute approximations to invariant sets in dynamical systems by minimizing an appropriate distance between a suitably selected finite set of points and its image under the dynamics. We demonstrate, through computational experiments, that this approach can successfully converge to approximations of (maximal) invariant sets of arbitrary topology, dimension, and stability, such as, e.g., saddle type invariant sets with complicated dynamics. We further propose to extend this approach by adding a Lennard-Jones type potential term to the objective function, which yields more evenly distributed approximating finite point sets, and illustrate the procedure through corresponding numerical experiments. Published by AIP Publishing.

[http://dx.doi.org/10.1063/1.4983468]
\end{abstract}

In the phase space of any nonlinear dynamical system, the "skeleton" of the global dynamical behavior consists of the invariant sets of the system, e.g., fixed points, periodic orbits, general recurrent sets, and the connecting orbits/invariant manifolds between them. Computational methods for approximating invariant sets have been, and will continue to be, a major part of the "toolkit" of every dynamical systems researcher, whether on the mathematical or on the modeling side. In this contribution we devise and implement a new variational approach for this task, which is able to compute invariant sets of arbitrary dimension, topology, and stability type. In addition-and in contrast to classical techniques-our method provides an approximate parametrization of the invariant set, which can be (smoothly) followed in parameter space.

\section{INTRODUCTION}

One central question in dynamical systems theory is to understand the existence and structure of invariant sets. Basic and important examples for invariant sets are fixed points/equilibria, periodic and quasiperiodic orbits and their associated stable, and unstable manifolds. In systems with chaotic behaviour, invariant sets with complicated topology may exist. A plethora of numerical techniques has been developed in order to approximate these sets computationally: Straightforward simulations (or more generally indirect methods) typically reveal parts of some invariant sets, e.g., some attractor or repeller of the system, cf., e.g., Ref. 20. They can also be adapted in order to compute saddle type invariant sets, cf., e.g., Refs. 13, 19, 17, and 21. Direct meth$o d s$, in contrast, focus on invariant sets of some particular type or topology like the examples mentioned above,

\footnotetext{
a) oj@tum.de

b)yannis@princeton.edu
}

including invariant sets of saddle type. They, however, include knowledge about the structure of the invariant set into the design of the method, in particular, on how to properly parametrize the set, cf., e.g., Refs. 1-3, 14, 15, 11, and 5 . In contrast, set oriented techniques are capable of approximating the invariant set without any a priori knowledge of its structure. ${ }^{7-10}$ In these, the set under consideration is covered by a subset of a (possibly cubical, as in GAIO) finite granularity decomposition of phase space. While this box covering provides a rigorous outer approximation to some invariant set, it does not provide a parametrization which varies smoothly in case that the invariant set varies smoothly with some system parameter. Rather, any change in the parametrization will come quantized (by the cube size). Consequently, we cannot quantify stability of the invariant set via infinitesimal concepts like spectral properties of some suitable operator.

The approach described in this paper is motivated by the desire to compute approximations to invariant sets of arbitrary topology, dimension, and stability type which do vary smoothly as mentioned. We propose to approximate some invariant set by a finite scattered point cloud which minimizes a certain objective functional (cf. Ref. 4 for another variational approach based on the lifetime of trajectories). In its most basic form, this functional is simply the distance (given by some metric on sets, as e.g., the Hausdorff metric) between the point cloud and its image under the dynamics. We give computational evidence that already this basic approach yields a useful approximation if the invariant set is (sufficiently strongly) hyperbolic. We further propose to augment this basic functional by a second term which penalizes a "too uneven" distribution of the point cloud. Here, we use a Lennard-Jones potential for this purpose. Our numerical experiments suggest that this indeed improves the approximation quality if the involved algorithm parameters are chosen appropriately. 


\section{INVARIANT SETS}

We consider a discrete-time dynamical system

$$
x_{k+1}=f\left(x_{k}\right), \quad k=0,1,2, \ldots,
$$

where $f: \mathbb{R}^{d} \rightarrow \mathbb{R}^{d}$ is a diffeomorphism (e.g., an explicit mapping or the time-T-map of some ordinary differential equation). A set $X \subset \mathbb{R}^{d}$ is invariant if

$$
X=f(X) \text {. }
$$

Simple examples for invariant sets are fixed points $\bar{x}=f(\bar{x})$ or periodic orbits $X=\left\{x_{0}, \ldots, x_{p-1}\right\}, x_{k+1} \bmod p=f\left(x_{k}\right)$. If $X \subset \mathbb{R}^{d}$ is invariant, then, by continuity of $f$, its closure is invariant as well, and so in the following, we can restrict our considerations to closed invariant sets. In fact, we will be concerned with compact invariant sets only. Given some compact set $Q \subset \mathbb{R}^{d}$, the set

$$
\operatorname{Inv}(Q)=\left\{x \in Q \mid f^{k}(x) \in Q \text { for all } k \in \mathbb{Z}\right\}
$$

is the maximal invariant set within $Q$. By definition, it contains all invariant sets which are contained in $Q$. In many cases, e.g., in the numerical experiments below, $\operatorname{Inv}(Q)$ is independent of $Q$ if $Q$ is chosen large enough.

\section{A VARIATIONAL SCHEME FOR INVARIANT SETS}

Our approach to computing compact invariant sets will be based on minimizing the distance between some compact set $X \subset \mathbb{R}^{d}$ and its image $f(X) \subset \mathbb{R}^{d}$. Let $\mathcal{C}$ be the set of non-empty compact subsets of $\mathbb{R}^{d}$, and let $d: \mathcal{C} \times \mathcal{C} \rightarrow$ $[0, \infty)$ be a metric on $\mathcal{C}$. Then,

$$
X=f(X) \quad \text { if and only if } \quad d(X, f(X))=0 .
$$

In any numerical computation, we can only work on some subset of $\mathcal{C}$ which can be described by finitely many parameters. In this subset, we cannot expect to satisfy $d(X, f(X))=0$. The idea of our approach is to minimize the ("energy") functional $E: \mathcal{C} \rightarrow[0, \infty)$,

$$
E(X)=d(X, f(X)),
$$

on some suitable subset $\tilde{\mathcal{C}} \subset \mathcal{C}$ instead.

Let $B_{r}(0) \subset \mathbb{R}^{d}$ be the ball centered at 0 with radius $r$, and recall that the subset relation $\subseteq$ is a partial order on $\mathcal{C}$.

Proposition 1. Suppose that $\operatorname{Inv}\left(B_{r}(0)\right)=S$ for some $S \in$ $\mathcal{C}$ for all sufficiently large $r$. Then, the set $S$ is the unique minimizer of $E$ on $\mathcal{C}$ which is maximal with respect to the subset relation.

Proof. By (1) and the definition of $E$, any minimizer of $E$ is an invariant set. Thus, the union $U=\cup_{X \in \mathcal{C}, X=f(X)} X$ of all compact invariant sets is a minimizer. Further, since it contains all other minimizers from $\mathcal{C}$, it is the unique set which is maximal with respect to the subset relation.

\section{A. The Hausdorff metric}

A common way to measure distances between compact sets is via the Hausdorff metric which is defined as follows:
For any non-empty set $X \subset \mathbb{R}^{d}$, the distance of a point $y \in$ $\mathbb{R}^{d}$ from $X$ is

$$
d(y, X)=\inf _{x \in X}\|y-x\|_{2}
$$

The distance of a second non-empty set $Y \subset \mathbb{R}^{d}$ from $X$ is

$$
d(Y, X)=\sup _{y \in Y} d(y, X),
$$

and since this distance is not symmetric, one defines the Hausdorff metric

$$
\begin{aligned}
d_{H}(X, Y) & =\max \{d(Y, X), d(X, Y)\} \\
& =\max \left\{\sup _{y \in Y} \inf _{x \in X}\|y-x\|_{2}, \sup _{x \in X} \inf _{y \in Y}\|y-x\|_{2}\right\}
\end{aligned}
$$

between $X$ and $Y$. Note that $\left(\mathcal{C}, d_{H}\right)$ is complete.

\section{B. A modified Hausdorff metric}

As mentioned, we are going to minimize the energy functional (2) on some subset of $\mathcal{C}$. In fact, we will simply use finite subsets $\tilde{X}=\left\{x_{1}, \ldots, x_{n}\right\} \subset \mathbb{R}^{d}$ (i.e., point clouds) for this purpose, such that $E$ can be seen as a function on $\mathbb{R}^{n d}$, where $n$ is the (fixed) number of points in these subsets. Unfortunately, $E$ : $\mathbb{R}^{\text {nd }} \rightarrow[0, \infty)$ is not smooth and this prevents us from using standard schemes for the minimization. We therefore employ the following modified Hausdorff distance instead. We use

$$
\hat{d}(y, \tilde{X})=d(y, \tilde{X})^{2}=\min _{x \in \tilde{X}}\|y-x\|_{2}^{2}
$$

in order to measure the distance of some point $y \in \mathbb{R}^{d}$ from some non-empty finite set $\tilde{X}$. We further define the distance

$$
\hat{d}(\tilde{Y}, \tilde{X})=\frac{1}{|\tilde{Y}|} \sum_{y \in \tilde{Y}} \hat{d}(y, \tilde{X})=\frac{1}{|\tilde{Y}|} \sum_{y \in \tilde{Y}} \min _{x \in \tilde{X}}\|y-x\|_{2}^{2}
$$

of some non-empty finite set $\tilde{Y}$ from $\tilde{X}(|\tilde{Y}|$ denotes the number of points in $\tilde{Y}$ ) and finally define the Hausdorff-like distance

$$
\hat{d}_{H}(\tilde{X}, \tilde{Y})=\frac{1}{2}(\hat{d}(\tilde{X}, \tilde{Y})+\hat{d}(\tilde{Y}, \tilde{X}))
$$

between two non-empty finite sets $\tilde{X}$ and $\tilde{Y}$. Note that $\hat{d}_{H}$ is a metric on the set of non-empty finite subsets of $\mathbb{R}^{d}$. For some set $\tilde{X}=\left\{x_{1}, \ldots, x_{n}\right\} \subset \mathbb{R}^{d}$, the corresponding energy functional reads explicitly

$$
\begin{aligned}
\hat{E}\left(x_{1}, \ldots, x_{n}\right)= & E(\tilde{X})=\hat{d}_{H}(\tilde{X}, f(\tilde{X})) \\
= & \frac{1}{2 n} \sum_{i=1}^{n} \min _{j=1: n}\left\|x_{i}-f\left(x_{j}\right)\right\|_{2}^{2} \\
& +\frac{1}{2 n} \sum_{i=1}^{n} \min _{j=1: n}\left\|f\left(x_{i}\right)-x_{j}\right\|_{2}^{2} \\
= & \frac{1}{2 n} \sum_{i=1}^{n}\left\|x_{i}-f\left(x_{j(i)}\right)\right\|_{2}^{2} \\
& +\frac{1}{2 n} \sum_{i=1}^{n}\left\|f\left(x_{i}\right)-x_{j(i)}\right\|_{2}^{2},
\end{aligned}
$$

where $j(i)=\operatorname{argmin}_{j=1, \ldots, n}\left\|x_{j}-f\left(x_{i}\right)\right\|^{2}$. 
Remark 1. Note that $\hat{E}(\tilde{X})=0$ if and only if $\tilde{X}$ is a union of periodic orbits, and one might expect this to prevent our approach from being able to compute, e.g., heteroclinic orbits. Since, however, in practice, any minimization scheme for $\hat{E}$ will be terminated as soon as some stopping criterion is fulfilled (e.g., when $\|\nabla \hat{E}(\tilde{X})\|<10^{-6}$ ), this does not seem to be an issue-cf. Experiment 5 where we cover part of the unstable manifold of a saddle type fixed point.

\section{Implementation}

We are going to minimize the energy functional $\hat{E}$ by a standard Quasi-Newton scheme, namely the limited memory Broyden-Fletcher-Goldfarb-Shanno (BFGS) scheme as implemented in the Matlab function fminlbfgs (by DirkJan Kroon, University of Twente). In order to compute the distance $\hat{d}(y, X)$ of some point $y$ from some finite set $\tilde{X}$, we employ a kd-tree based search for some point $x=x(y) \in \tilde{X}$ which is closest to $y$. This is conveniently implemented in the knnsearch command in Matlab. In fact, knnsearch can return the $k \in \mathbb{N}$ nearest neighbours at once and each query of this type takes $\mathcal{O}(\log |\tilde{X}|)$ time. Overall, this translates into a running time of $\mathcal{O}(|\tilde{X}| \log (\tilde{X}))$ and all the examples in Sec. IV only take a few seconds to run on a recent machine. For $|\tilde{X}|=10^{4}$, the runtime will be a few minutes.

\section{COMPUTATIONAL EXPERIMENTS}

Experiment 1. (Fixed point in 1d) Let us start with the simplest possible example: a linear map on the line. We consider $f: \mathbb{R} \rightarrow \mathbb{R}, f(x)=a x$ with $a=0.1$ and $a=10$. The maximal invariant set in $Q=[-1,1]$ is $\operatorname{Inv}(Q)=\{0\}$. We initialize $\tilde{X}$ with 40 points, chosen randomly from $[-1$, 1] according to a uniform distribution and terminate the BFGS iteration as soon as $\|\nabla \hat{E}\|_{\infty}<10^{-6}$. Figure 1 shows the evolution of $\tilde{X}$ in course of the optimization for both values of $a$. The BFGS iteration terminates after 21 and 18 steps with an $\hat{E}$ value of around $10^{-11}$, and the Hausdorff distance of $\tilde{X}$ from $\{0\}$ is $\approx 10^{-6}$ for $a=0.1$ and $\approx 2 \times$ $10^{-5}$ for $a=10$.

The speed of convergence seems to strongly depend on the contraction constant $a$ : Figure 2 shows the evolution of $\tilde{X}$ in course of the BFGS iteration for $a=1.1$ (left) and $a=1.01$ (right). While in both cases the objective function value is less than $10^{-8}$, the Hausdorff distance of $\tilde{X}$ from $\{0\}$ is still rather large, namely $\approx 0.003$ for $a=1.1$ and $\approx 0.1$ for $a=1.01$, even after a much larger number of iterations.

Experiment 2. (A connecting orbit in 1d) We next consider a nonlinear map on the line for which the maximal invariant set is the interval $[0,1]$ and the map is $f(x)=x+a x(1-x)$ with $a=0.8$. It possesses two fixed points, namely $\bar{x}_{1}=0$ (unstable) and $\bar{x}_{2}=1$ (stable). Points within $(0,1)$ are heteroclinic to these two equilibria so that the maximal invariant set within any set $Q$ covering $[0,1]$ is the interval $[0,1]$. We choose $Q=[-1,2]$ and initialize $X$ by a set of points chosen randomly from $[-1,2]$ according to a uniform distribution. Figure 3 shows the evolution of $\tilde{X}$ in course of the BFGS iteration for $n=100$ (left), as well as the Hausdorff distance $d_{H}$ between $\tilde{X}$ and $[0,1]$ (approximated by computing $d_{H}$ between $\tilde{X}$ and a grid of $10^{4}$ points in $\left.[0,1]\right)$.

Experiment 3. (A connecting orbit in 2d) Similarly, for the map

$$
f(x, y)=\left(1.5 x^{3}-0.5 x, 10 y\right)
$$

with fixed points $(-1,0)$ (unstable center), $(0,0)$ (saddle), and $(1,0)$ (unstable center), the maximal invariant set within any set $Q$ which contains $[-1,1] \times\{0\}$ is $\operatorname{Inv}(Q)=[-1$, $1] \times\{0\}$. We start with a set $\tilde{X}$ of 100 points which are chosen randomly from $[-2,2]^{2}$ according to a uniform distribution. Figure 4 shows the iterates of $\tilde{X}$ in course of the optimization after 3 and 30 BFGS steps.

Experiment 4. (An unstable invariant disk in the plane) We repeat the experiment with a map for which the maximal invariant set inside a sufficiently large neighborhood is an unstable disk. We consider the vector field

$$
v(x, y)=\left[\begin{array}{c}
-y+a x\left(x^{2}+y^{2}-1\right) \\
x+a y\left(x^{2}+y^{2}-1\right)
\end{array}\right]
$$

with $a=10$ and define the map $f$ as one Euler step with step size $h=0.1$, i.e.,

$$
f(x, y)=(x, y)+h v(x, y)
$$

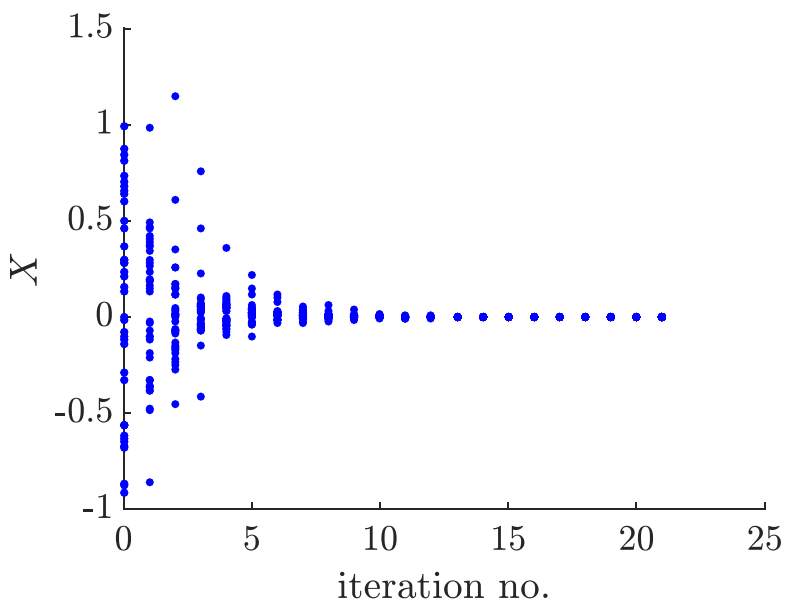

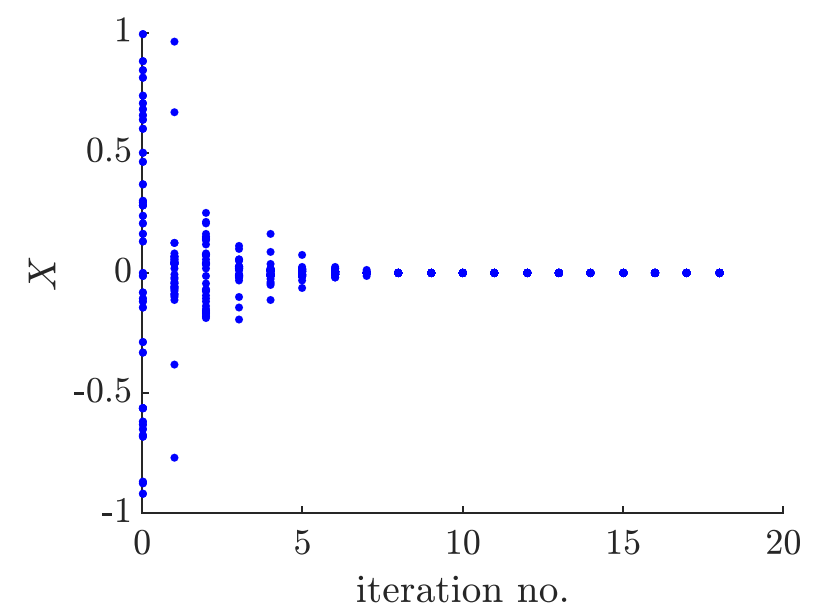

FIG. 1. Linear map on the line, evolution of $\tilde{X}$ in course of the optimization. Left: $a=0.1$; right: $a=10$. 

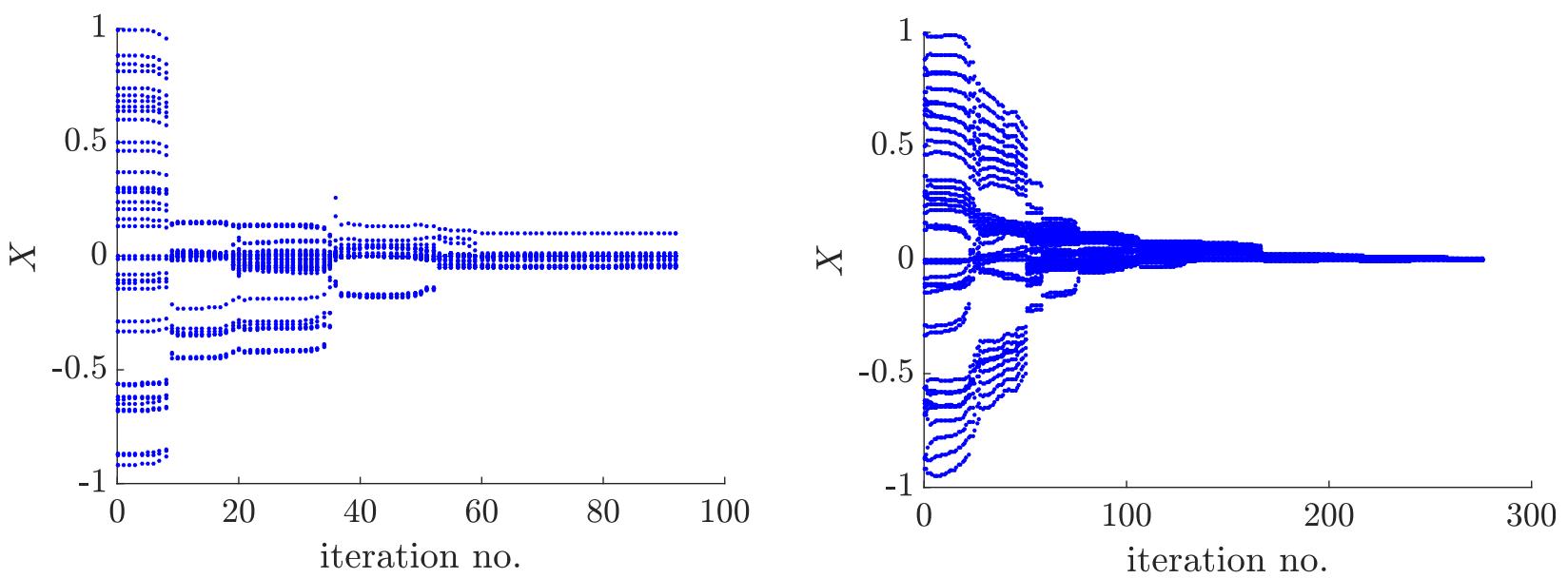

FIG. 2. Linear map on the line, evolution of $\tilde{X}$ in course of the optimization. Left: $a=1.1$; right: $a=1.01$.
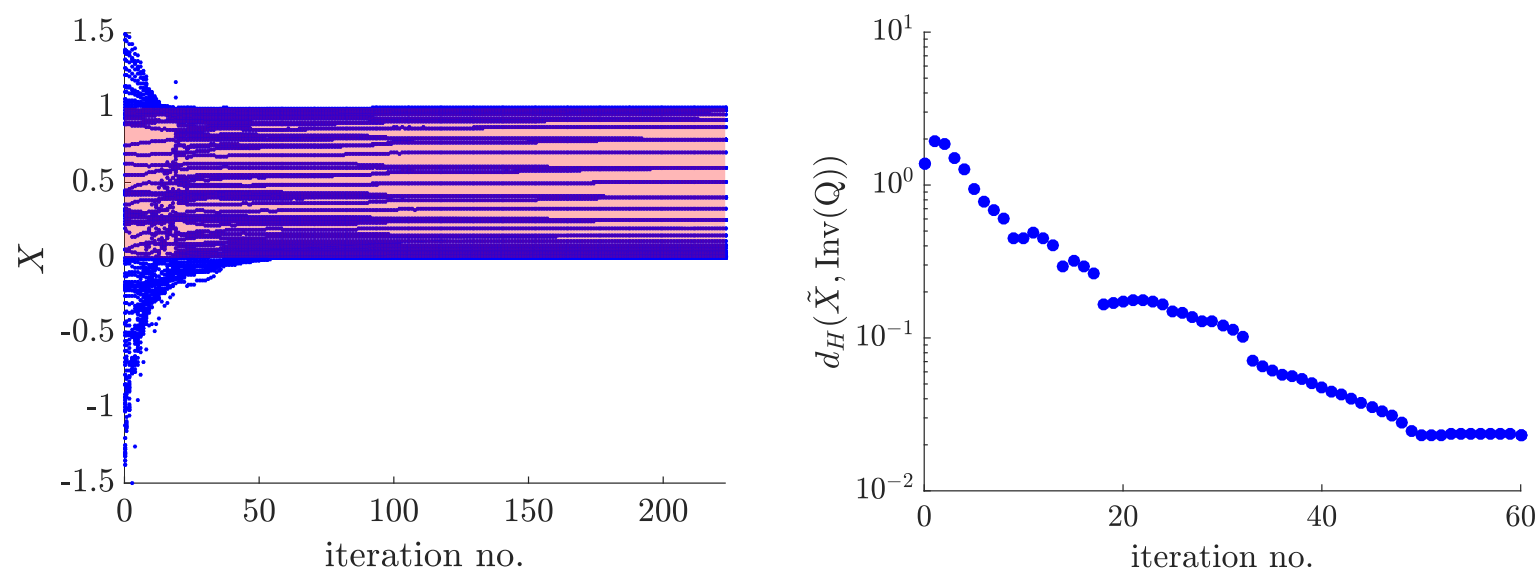

FIG. 3. Connecting orbit on the line: approximation of $\operatorname{Inv}(Q)=[0,1]$ (lightly red shaded) by a set $\tilde{X}$ of $n=100$ (left) points. Right: the Hausdorff distance between $\tilde{X}$ and the maximal invariant set $[0,1]$ shrinks to $\approx 3 \times 10^{-2}$ in course of the iteration.
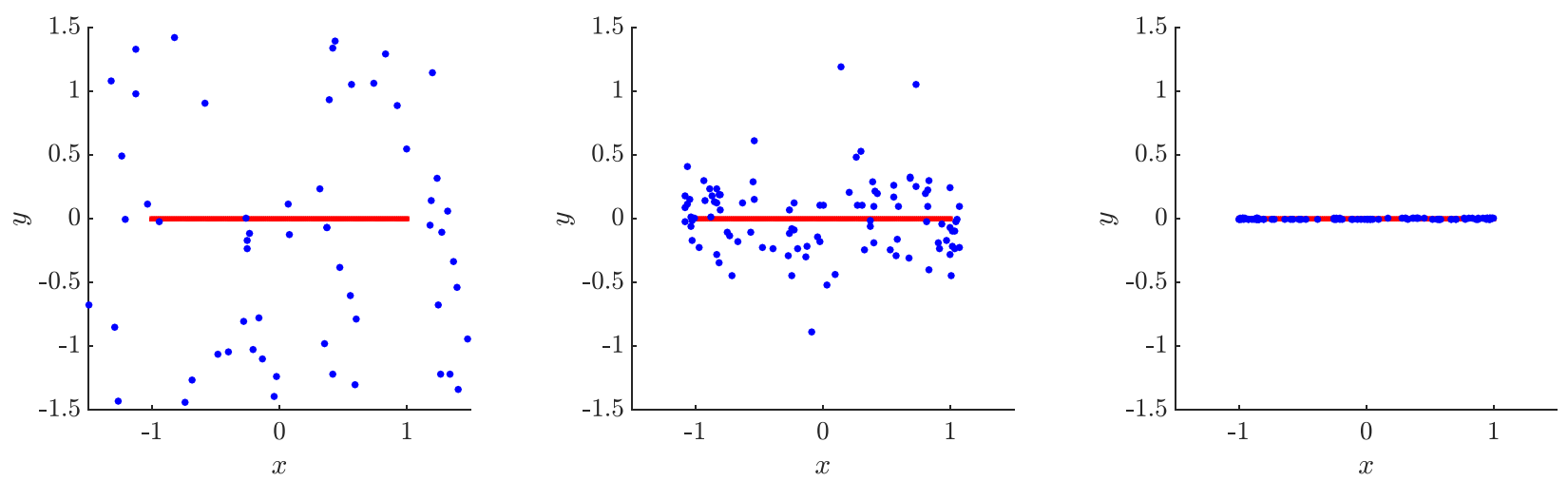

FIG. 4. Connecting orbit in the plane: the set $\tilde{X}$ (blue) initially (left) and after 3 (center) and 30 (right) BFGS iterations.

We start with a set $\tilde{X}$ of 1000 points which are chosen randomly from $[-2,2]^{2}$ according to a uniform distribution. Figure 5 shows the iterates of $\tilde{X}$ in course of the optimization after 3 and 30 BFGS steps.

Clearly, the objective function $\hat{E}$ will typically possess many local minima, and the result of the minimization will strongly depend on the initialization of $\tilde{X}$. This is exemplified in Fig. 6, where the results of the BFGS after 500 iterations are shown for different initializations of $\tilde{X}$. This is one motivation for the construction proposed in Sec. V.
Experiment 5. (The Hénon map) Let us now consider an example with a chaotic invariant set as exhibited by the Hénon map

$$
f(x, y)=\left(1-a x^{2}+y, b x\right) .
$$

For $a=1.2, b=0.3$, this map possesses an attractor as shown in Fig. 7 (left). The maximal invariant set [in the center of that figure, computed by GAIO (https://github.com/gaioguy/ GAIO $)^{7}$ ] additionally contains a saddle fixed point around 

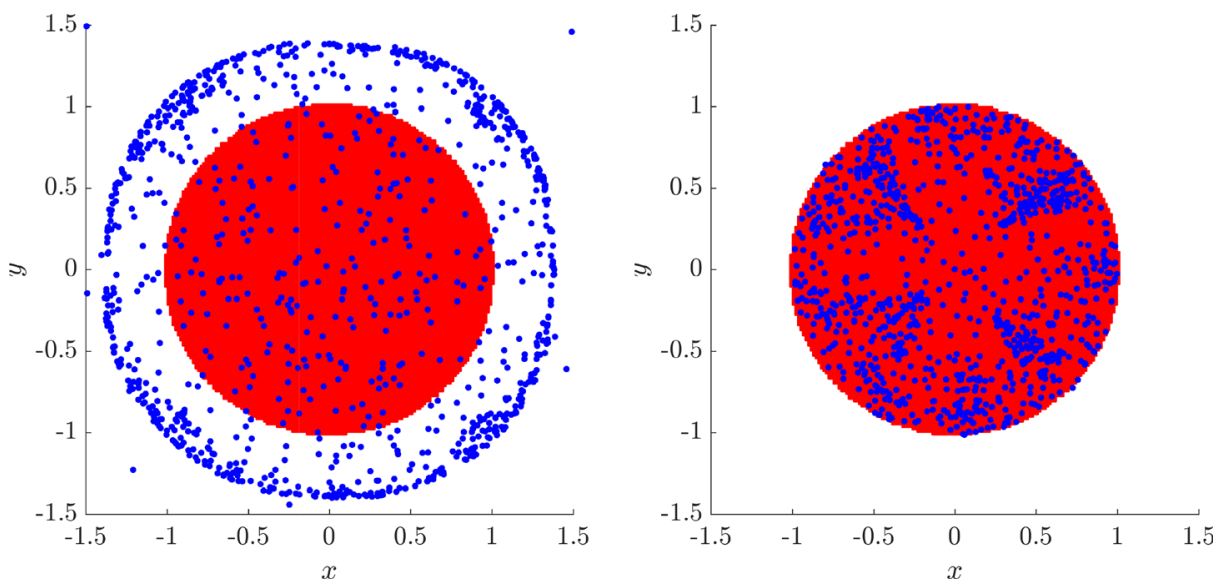

FIG. 5. Unstable invariant disk (red) in the plane: the set $\tilde{X}$ (blue) after 3 (left) and 30 (right) BFGS iterations.
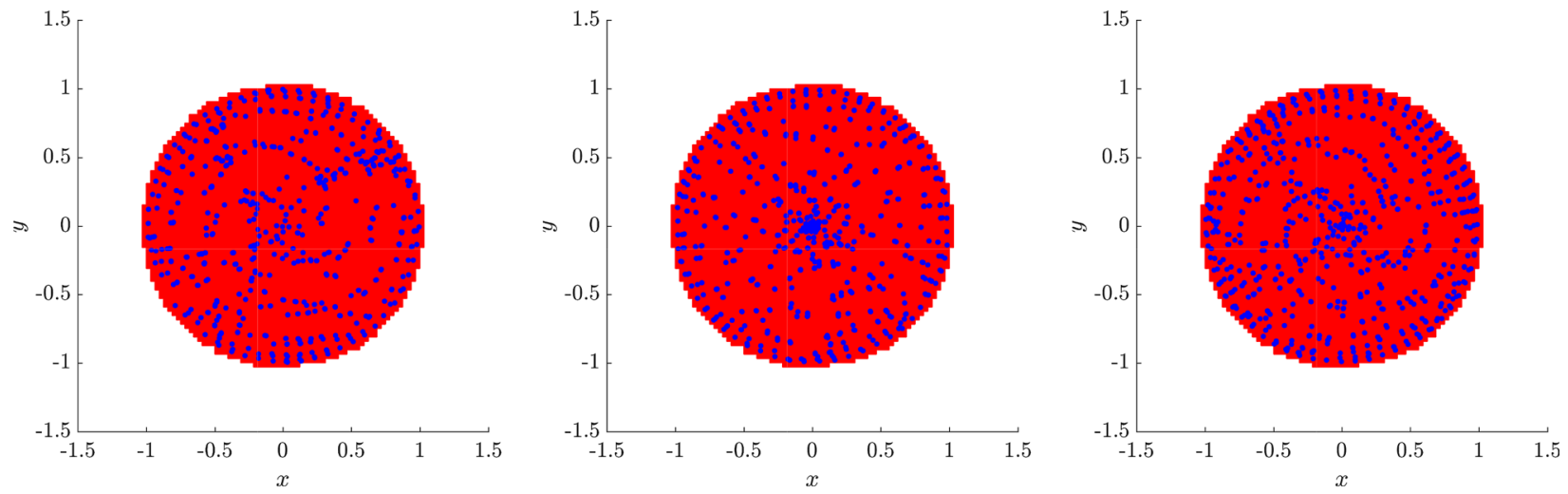

FIG. 6. Unstable invariant disk (red) in the plane: the 500th iterate of $\tilde{X}$ for an initial set $\tilde{X}$ of 1000 points chosen from a uniform grid (left), randomly (center), and as pseudo-random points (right), i.e., Halton points. ${ }^{18}$

$(-1.25,-1.1)$ and part of its unstable manifold connecting the fixed point to the attractor. The point cloud that results from our variational approach approximately fills this covering. We initialized the optimization with a set $\tilde{X}$ of points which have been chosen randomly from the square $[-2,2]^{2}$ according to a uniform distribution and ran the BFGS scheme until the value of the objective function fell below $10^{-6}$. Clearly, there are regions (surrounded by black lines) where points converge extremely slowly. We conjecture that this is due to (near-)tangencies between stable and unstable manifolds, i.e., (near-)nonhyperbolic behaviour.
We further consider the case $a=2$ where the map possesses a saddle type maximal invariant set with a Cantor-like structure, cf. Ref. 19. Note, in particular, that this set cannot be computed/observed by mere simulation in forward or backward time since this set is unstable in both time directions (this is the unicorn we are alluding to in the title). Figure 8 shows a covering of the maximal invariant set as well as its approximation by 1000 points through our variational scheme after 20 and 200 steps of the optimizer, as well as after the gradient of the objective function decreased below $10^{-6}$.
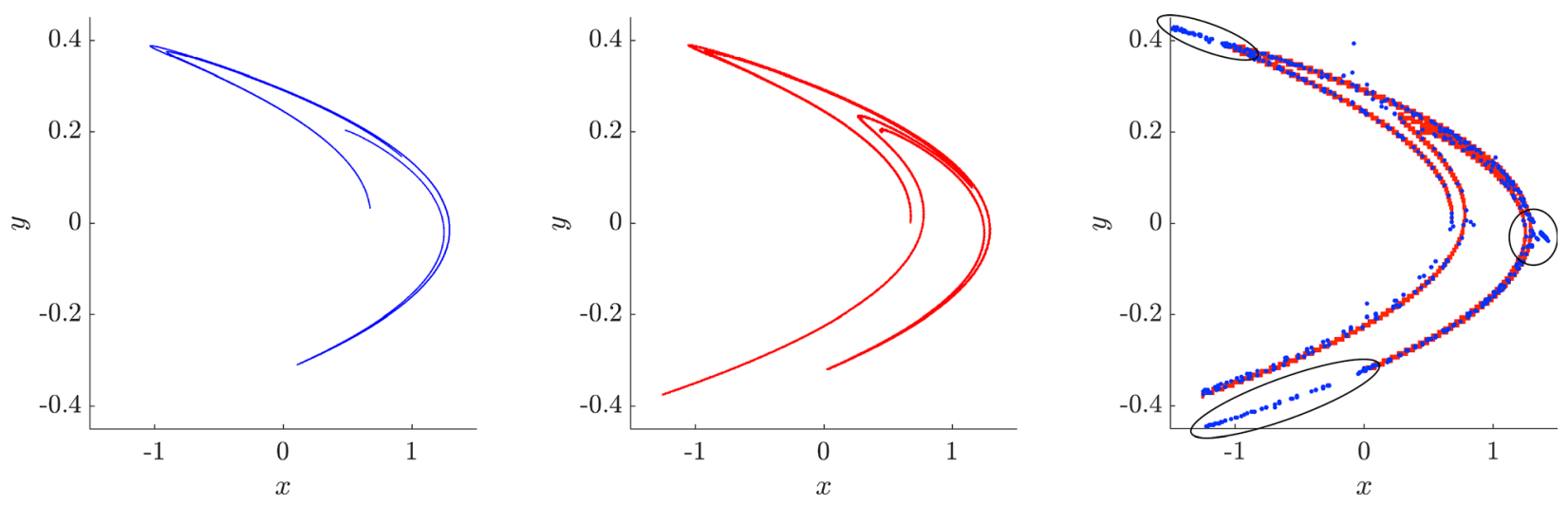

FIG. 7. Hénon map: attractor (left), maximal invariant set (center), and the iterates of 1000 randomly chosen points after termination of the BFGS scheme (when $|\hat{E}(\tilde{X})|<10^{-6}$ after 769 steps). 

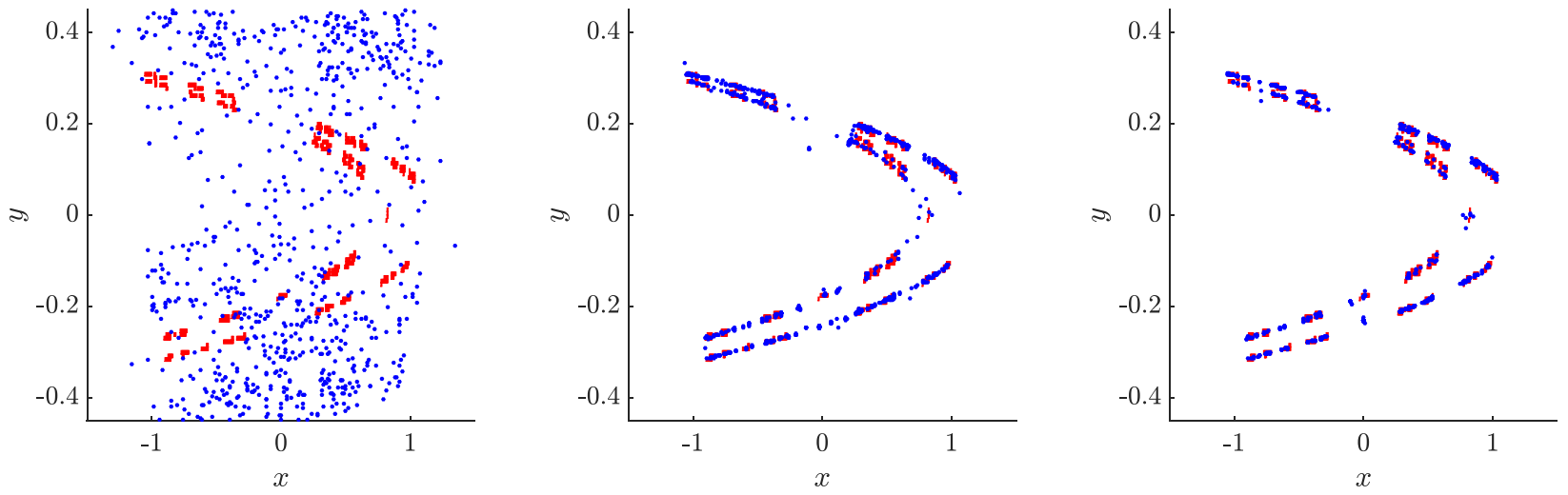

FIG. 8. Maximal invariant set in the Hénon map: iterates of an initial set of 1000 randomly chosen points after 20 (left) and 200 (center) steps of the BFGS scheme, as well as after termination $\left(\|\nabla \hat{E}\|_{\infty}<10^{-6}, 928\right.$ steps $)$.

Experiment 6. (A chaotic saddle in 3d) We finally consider an example in $\mathbb{R}^{3}$ exhibiting a saddle type maximal invariant set with complicated dynamics and fractal structure. The map is $f(x, y, z)=\left(y, z, a+b x+c y-z^{2}\right)$ with $a=2.2, b=0.1$, and $c=0.3$, which is constructed in analogy to the Hénon map. Figure 9 shows a covering of the maximal invariant set within the cube $[-2,2]^{3}$ computed by GAIO. We initialize the optimization of $\hat{E}$ with a set $\tilde{X}$ of 500 points which have been chosen randomly from the cube $[-2,2]^{3}$ according to a uniform distribution. Figure 10 shows the iterates of $\tilde{X}$ after 20 and 200 steps of the optimizer, as well as after termination. Again, we observe slow convergence in certain regions like in the $2 \mathrm{~d}$ Hénon example.

\section{ADDITIONAL POTENTIALS}

While the points in $\tilde{X}$ seem to converge towards the maximal invariant $\operatorname{set} \operatorname{Inv}(Q)$ in the experiments above, their distribution is typically far from uniform on it. Moreover, one seems to obtain different minimizers depending on the initialization (and also we do not identify vectors which yield the same set, i.e., we do not factor by the permutational symmetries of the vector). In fact, in many of the experiments,

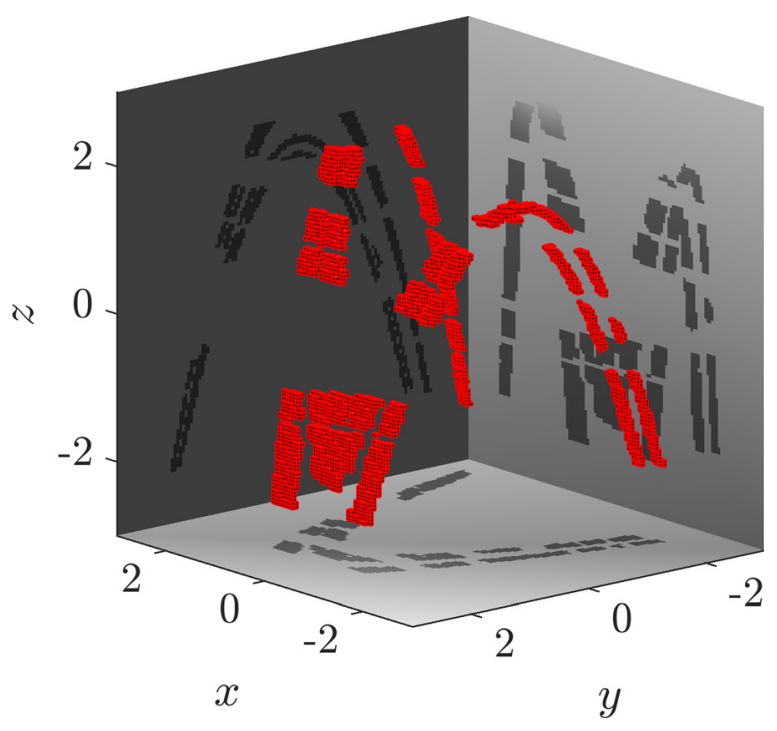

FIG. 9. 3d chaotic saddle: box covering of the maximal invariant set. points tend to cluster quite heavily in certain areas and even coincide (cf. Fig. 6). In view of our goal to best approximate the maximal invariant set in terms of the Hausdorff distance and to ultimately obtain a unique minimizer, it would be desirable to distribute $\tilde{X}$ more uniformly.

As a first step towards this goal, we are going to add a term to the potential $\hat{E}$, which strongly penalizes points in $\tilde{X}$ from getting too close and favors them to attain a certain distance $\delta$ between each other. This can be accomplished by a Lennard-Jones potential, cf. Ref. 12,

$$
V_{\delta}(r)=\left(\frac{\delta}{r}\right)^{2 p}-2\left(\frac{\delta}{r}\right)^{p}+1,
$$

where the exponent $p \in \mathbb{N}$ controls the "rigidity" of the potential and where $r$ is the distance between two points in $\tilde{X}$. In the following experiments, $p=1$ seemed to work best for our purposes. The proper distance $\delta$ ultimately depends on the dimension of $\operatorname{Inv}(Q)$ and the number $n$ of points in $\tilde{X}$ so that we cannot fix the value of $\delta$ a priori and we therefore include $\delta$ as an optimization variable. One can imagine the Lennard-Jones potential to be a "soft" version of the hard sphere potential, ${ }^{12}$ and correspondingly, we here imagine the points in $\tilde{X}$ to be surrounded by balls of radius $\delta$.

For each point in $\tilde{X}$, we are going to restrict the evaluation of $V$ to the $m$ nearest points from $\tilde{X}$. The corresponding augmented objective function reads

$$
\begin{aligned}
J\left(x_{1}, \ldots, x_{n}, \delta\right)= & \hat{E}\left(x_{1}, \ldots, x_{n}\right) \\
& +\mu \frac{1}{n} \sum_{i=1}^{n} \frac{1}{m} \sum_{j \in N_{m}(i)} V_{\delta}\left(\left\|x_{i}-x_{j}\right\|_{2}\right),
\end{aligned}
$$

where $N_{m}(i)$ is the set of $m$ nearest neighbours of $x_{i}$ and $\mu>0$ is a weighting parameter. Larger $\mu$ will favor the points from $\tilde{X}$ to attain a lattice structure, while smaller $\mu$ favors them to be close to some invariant set.

\section{A. Computational experiments}

Experiment 7. We reconsider experiment 4, choose $\mu=1$, and initialize $\tilde{X}$ as a uniform grid of $n=32 \times 32=1024$ points within the square $Q=[-2,2]^{2}$. We initialize $\delta=\sqrt{m(Q) /(n \pi)}$, i.e., such that the sum of the 

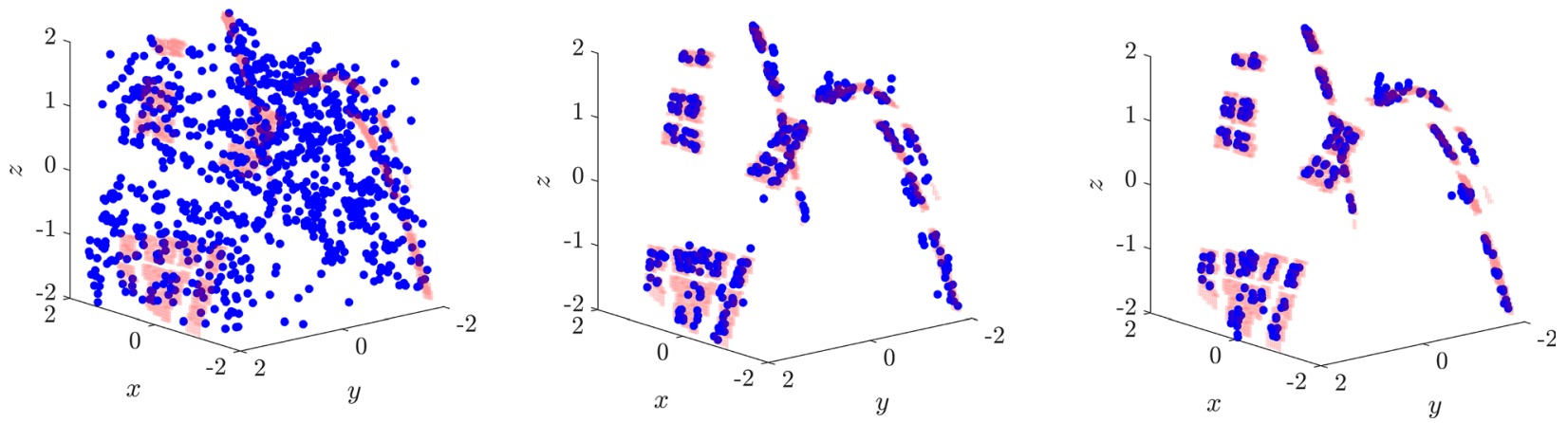

FIG. 10. 3d chaotic saddle: iterates of an initial set of 500 randomly chosen points after 20 (left) and 200 (center) steps of the BFGS scheme, as well as after termination $\left(\|\nabla \hat{E}\|_{\infty}<10^{-6}, 727\right.$ steps $)$.

volumes of balls centered at the points in $\tilde{X}$ with radius $\delta$ is of the same order as the volume of $Q$. Figure 11 shows the iterates of $\tilde{X}$ after 500 steps of the BFGS scheme for $m=6$ (left) and $m=30$ (right). The larger number of neighbors yields a much better approximation.

Figures 12 and 13 show the results of the same experiment, albeit for the Hénon map. While a larger number of neighbours tends to yield a more uniform covering of the maximal invariant set here as well, they also tend to hide finer structures (given a fixed number of balls).

\section{DISCUSSION AND FUTURE DIRECTIONS}

In the numerical experiments above, we have used the GAIO output as a reference for the computations since in principle it provides a rigorous covering of the maximal invariant set, cf. Ref. 6. While the approach proposed in this paper does not live up to this goal, it (1) potentially might be applicable to higher dimensional problems more efficiently since we do not need to map subsets of full state space dimension and (2) it potentially might be more suitable for a numerical study of bifurcations of invariant sets since the point cloud can be varied smoothly with a system parameter. A simple example would be the tracking of an attracting invariant circle through resonance horns. While the attractor changes from periodic to quasiperiodic depending on the rationality of the frequency ratio, the approximating point cloud would change smoothly since while the attractor changes, the maximal invariant set persists and varies smoothly.

Clearly, the experiments in this paper can only be seen as a first step. Of course, it would be desirable to gain insight into the general convergence behavior of the scheme, in particular as the number of points goes to infinity, and this is currently under investigation. Further, it would be desirable to alleviate the bad convergence behavior in weakly hyperbolic regions.

While the inclusion of the Lennard-Jones potential seems to point in the right direction, it also raises new issues like the proper number of neighbors and the proper value of the weighting parameter $\mu$. Of course, other potentials might be conceivable as well. In particular, it might be useful to adapt the "radius parameter" $\delta$ locally, i.e., use balls of smaller radius where appropriate. A multilevel scheme might be useful where one considers balls of several scales at the same time in the spirit of the famous "cheese theorem" of Lieb. ${ }^{16}$

As mentioned, in principle any metric on the set of compact subsets of $\mathbb{R}^{d}$ will do. Our choice of a Hausdorff type distance was motivated by smoothness considerations. A natural candidate for a different choice would be the Wasserstein or earth mover's distance (where $\tilde{X}$ is seen as a sum of atomic measures). We will explore whether this bears any advantage over the Hausdorff type distance used here (in
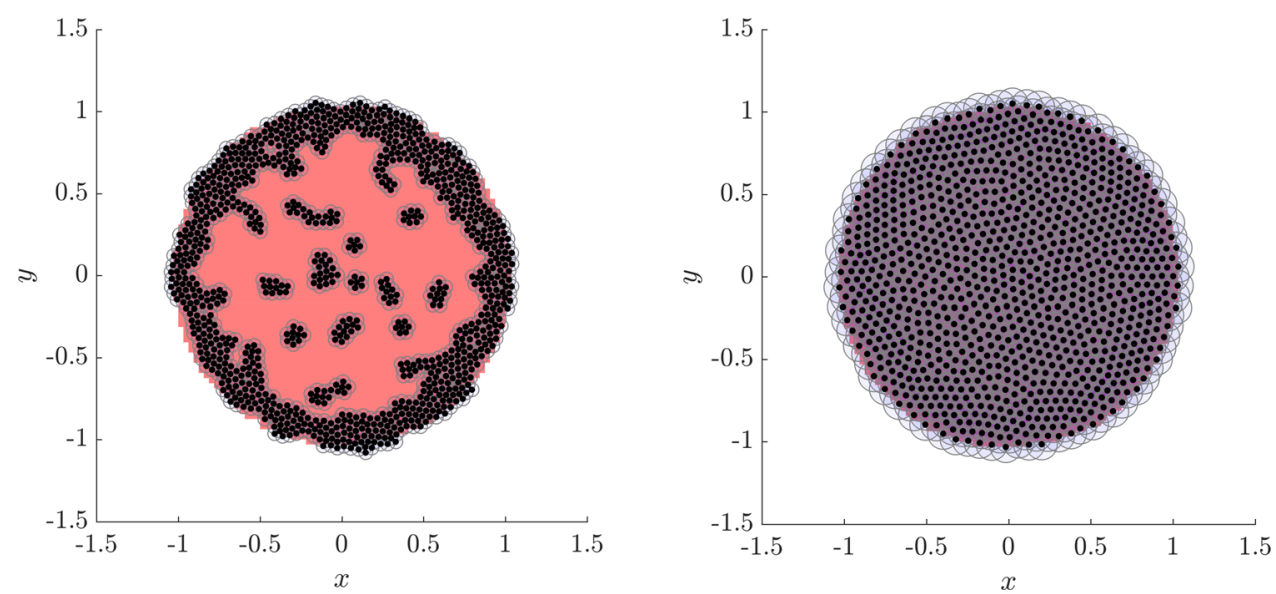

FIG. 11. Invariant disk, with Lennard-Jones potential: 500th iterate of the initial point cloud for $m=6$ (left) and $m=30$ (right). We show the set $\tilde{X}$ (black dots) together with surrounding balls of radius $\delta$ (where $\delta$ results from the optimization). 

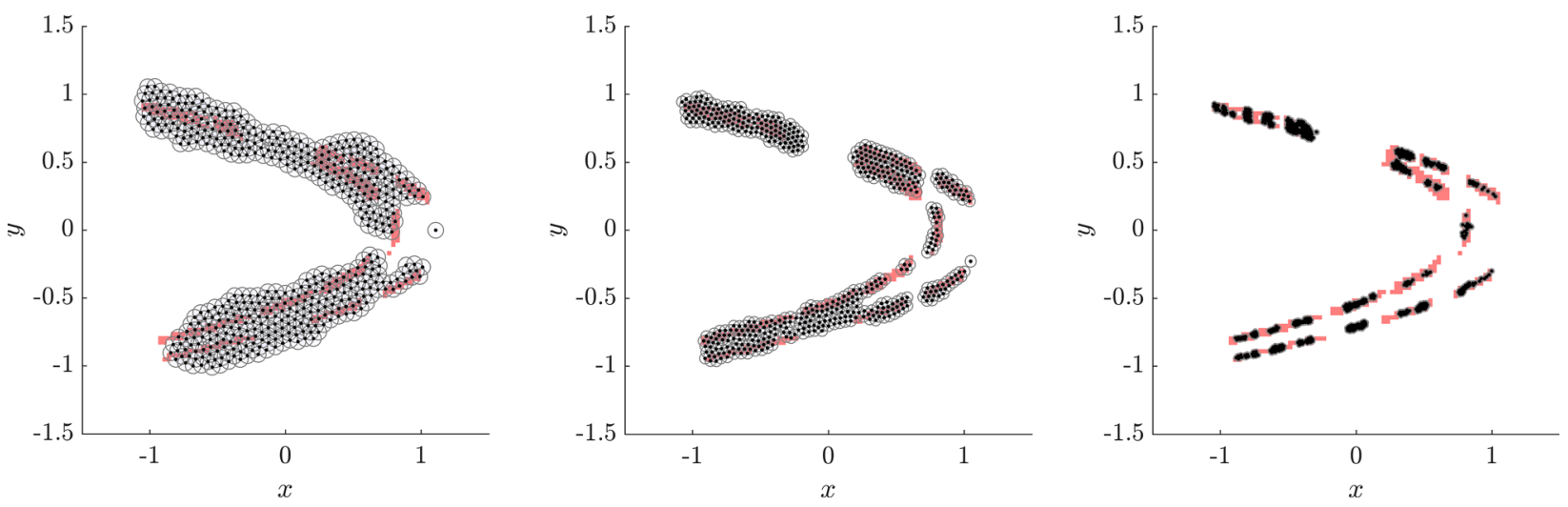

FIG. 12. Hénon map, with Lennard-Jones potential: 500th iterate of the initial point cloud for $m=6$ and $\mu=1,0.1$, and 0.01 (from left to right).
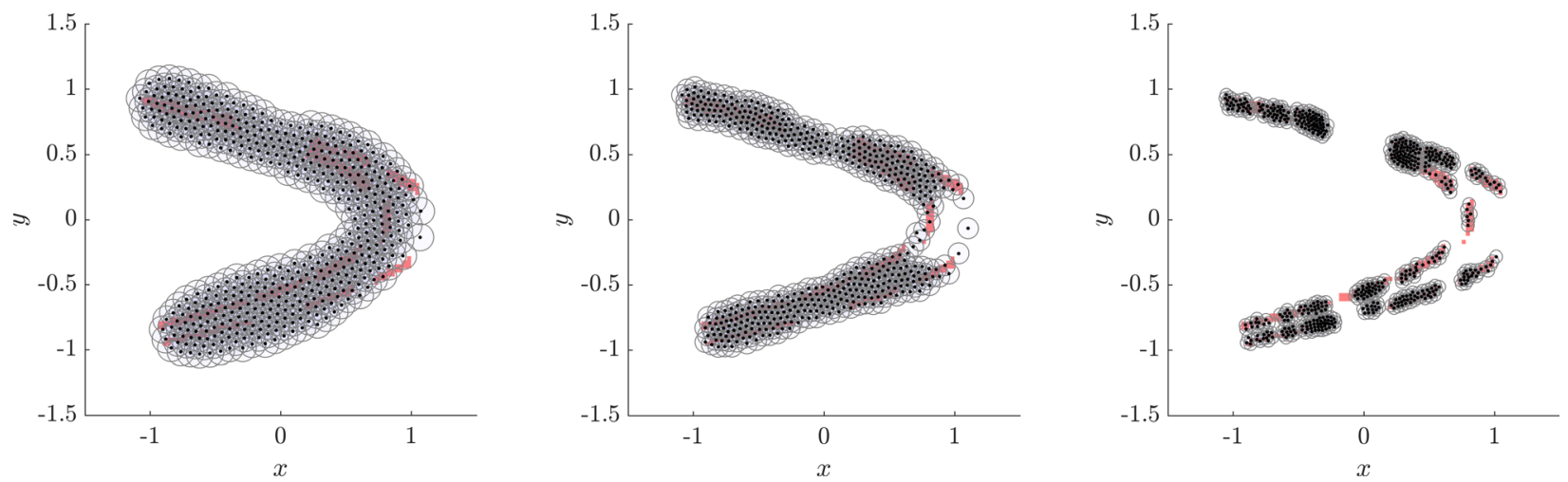

FIG. 13. Hénon map, with Lennard-Jones potential: 500th iterate of the initial point cloud for $m=30$ and $\mu=1,0.1$, and 0.01 (from left to right).

particular, since the numerical effort for computing the Wasserstein distance is presumably larger than that for the Hausdorff type metric).

With the limited memory BFGS scheme, we used a standard quasi-Newton method for the minimization of the objective functional. Depending on the set-metric employed, other schemes might be more beneficial, both from a theoretical point of view (in order to prove convergence) and also from a numerical efficiency point of view.

As mentioned, one of the motivations for considering the approach advocated in this paper was to construct an approximation of some invariant set which varies smoothly in the case that the underlying invariant set varies smoothly with some system parameter. In fact, it is an interesting question whether our approach can be embedded into a path following scheme.

Another interesting question is how to modify the functional $E$ such that an invariant set of particular type is computed, e.g., is it possible to directly compute the chain recurrent set instead of the maximal invariant one.

\section{ACKNOWLEDGMENTS}

We thank Gero Friesecke and William Leeb for helpful discussions as well as Daniel Karrasch for careful proofreading and helpful suggestions. We also gratefully acknowledge support of the Institute of Advanced Studies at the Technical University Munich.
O.J. was supported by the DFG Collaborative Research Center SFB/TR 109 "Discretization in Geometry and Dynamics." The work of I.G.K. is also partially supported by the U.S. National Science Foundation (CBET and CDS\&E). The support of the Institute for Advanced Study at TU Munich through a Hans Fischer Senior Fellowship to I. G. K. is gratefully acknowledged.

${ }^{1}$ R. Baier, M. Dellnitz, M. Hessel-von Molo, S. Sertl, and I. G. Kevrekidis, "The computation of convex invariant sets via Newton's method," J. Comput. Dyn. 1(1), 39-69 (2014).

${ }^{2}$ W.-J. Beyn, "Numerical methods for dynamical systems," in: Light W, ed. Advances in numerical analysis (Oxford science publications, Oxford, Clarendon Pr., 1991), pp. 175-236.

${ }^{3}$ W.-J. Beyn, A. Champneys, E. Doedel, W. Govaerts, Y. A. Kuznetsov, and B. Sandstede, "Numerical continuation, and computation of normal forms," in Handbook of Dynamical Systems (North-Holland, Amsterdam, 2002), Vol. 2, pp. 149-219.

${ }^{4} \mathrm{E}$. M. Bollt, "The path towards a longer life: On invariant sets and the escape time landscape," Int. J. Bifurcation Chaos Appl. Sci. Eng. 15(5), 1615-1624 (2005).

${ }^{5}$ K. A. Cliffe, A. Spence, and S. J. Tavener, "The numerical analysis of bifurcation problems with application to fluid mechanics," in Acta Numerica (Cambridge University Press, Cambridge, 2000), pp. 39-131.

${ }^{6} \mathrm{~S}$. Day, O. Junge, and K. Mischaikow, "A rigorous numerical method for the global analysis of infinite-dimensional discrete dynamical systems," SIAM J. Appl. Dyn. Syst. 3(2), 117-160 (2004).

${ }^{7}$ M. Dellnitz, G. Froyland, and O. Junge, "The algorithms behind GAIO set oriented numerical methods for dynamical systems," in Ergodic Theory, Analysis, and Efficient Simulation of Dynamical Systems, edited by B. Fiedler (Springer, Berlin, 2001), pp. 145-174, 805-807. 
${ }^{8}$ M. Dellnitz and A. Hohmann, "The computation of unstable manifolds using subdivision and continuation," in Nonlinear Dynamical Systems and Chaos (Groningen, 1995) (Birkhäuser, Basel, 1996), pp. 449-459.

${ }^{9}$ M. Dellnitz and A. Hohmann, "A subdivision algorithm for the computation of unstable manifolds and global attractors," Numer. Math. 75(3), 293-317 (1997).

${ }^{10} \mathrm{M}$. Dellnitz and O. Junge, "Set oriented numerical methods for dynamical systems," in Handbook of Dynamical Systems III: Towards Applications, edited by B. Fiedler, G. Ioos, and N. Kopell (World Scientific Publishing Co. Inc., Amsterdam, 2002), pp. 221-264.

${ }^{11}$ E. Doedel and B. E. Oldeman, AUTO-07P: Continuation and Bifurcation Software for Ordinary Differential Equations (Concordia University, 2009).

${ }^{12}$ J. P. Hansen and I. R. McDonald, Theory of Simple Liquids (Elsevier Science, 1990).

${ }^{13}$ H. Kantz and P. Grassberger, "Repellers, semi-attractors, and long-lived chaotic transients," Phys. D: Nonlinear Phenom. 17(1), 75-86 (1985).

${ }^{14}$ I. G. Kevrekidis, R. Aris, L. D. Schmidt, and S. Pelikan, "Numerical computation of invariant circles of maps," Phys. D: Nonlinear Phenom. 16(2), 243-251 (1985).
${ }^{15}$ B. Krauskopf and H. Osinga, "Globalizing two-dimensional unstable manifolds of maps,” Int. J. Bifurcation Chaos 8(3), 483-504 (1998).

${ }^{16} \mathrm{E}$. H. Lieb and J. L. Lebowitz, "The constitution of matter: Existence of thermodynamics for systems composed of electrons and nuclei," Adv. Math. 9(3), 316-398 (1972).

${ }^{17} \mathrm{P}$. Moresco and S. Ponce Dawson, "The PIM-simplex method: An extension of the PIM-triple method to saddles with an arbitrary number of expanding directions," Physica D 126(1-2), 38-48 (1999).

${ }^{18} \mathrm{H}$. Niederreiter, Random Number Generation and Quasi-Monte Carlo Methods (Society for Industrial and Applied Mathematics, 1992).

${ }^{19}$ H. E. Nusse and J. A. Yorke, "A procedure for finding numerical trajectories on chaotic saddles," Physica D 36(1-2), 137-156 (1989).

${ }^{20}$ A. M. Stuart and A. R. Humphries, Dynamical Systems and Numerical Analysis, Cambridge Monographs on Applied and Computational Mathematics Vol. 2 (Cambridge University Press, Cambridge, 1996).

${ }^{21}$ D. Sweet, H. E. Nusse, and J. A. Yorke, "Stagger-and-step method: Detecting and computing chaotic saddles in higher dimensions," Phys. Rev. Lett. 86(11), 2261-2264 (2001). 